\title{
THE
}

1996

\section{Acoustic scattering from a thermally driven buoyant plume}

John Oeschger

University of Rhode Island

Louis Goodman

Follow this and additional works at: https://digitalcommons.uri.edu/phys_facpubs

Terms of Use

All rights reserved under copyright.

\section{Citation/Publisher Attribution}

Oeschger, J., \& Goodman, L. (1996). Acoustic scattering from a thermally driven buoyant plume. Journal of the Acoustical Society of America, 100(3), 1451-1462. doi: 10.1121/1.415992.

Available at: https://doi.org/10.1121/1.415992

This Article is brought to you for free and open access by the Physics at DigitalCommons@URI. It has been accepted for inclusion in Physics Faculty Publications by an authorized administrator of DigitalCommons@URI. For more information, please contact digitalcommons-group@uri.edu. 


\section{Acoustic scattering from a thermally driven buoyant plume}

J. Oeschger, and L. Goodman

Citation: The Journal of the Acoustical Society of America 100, 1451 (1996); doi: 10.1121/1.415992

View online: https://doi.org/10.1121/1.415992

View Table of Contents: http://asa.scitation.org/toc/jas/100/3

Published by the Acoustical Society of America 


\section{Acoustic scattering from a thermally driven buoyant plume}

J. Oeschger ${ }^{\mathrm{a})}$

Department of Physics, University of Rhode Island, Kingston, Rhode Island 02881

L. Goodman

Office of Naval Research, Ocean and Atmospheric Physics Division, 800 N. Quincy Street, Arlington, Virginia 22217

(Received 6 February 1995; accepted for publication 15 September 1995)

An examination is made of the use of broad bandwidth high-frequency acoustic scattering to infer remotely the spatial structure of the temperature field of a thermally driven buoyant plume. Application of the far-field Born approximation results in a linear relationship between the transfer function of the scattering process, $\Gamma$, the ratio of received to transmitted pressure, to the spatial Fourier transform of the temperature field, $\phi(K)$, where $\mathbf{K}=\mathbf{k}_{s}-\mathbf{k}_{i}$ is the Bragg wave-number vector. A series of experiments are devised to test this hypothesis. These experiments involve a geometry of scattering in which pairs of sources and receivers are placed on opposite sides and equidistant from the scattering volume, a buoyant plume generated by a small circular heating element at the base of a water tank. It is shown that the far-field approximation assuming incident plane waves breaks down when the scales of temperature variability of the plume are of order the Fresnel radius. These results are discussed for both an unstable and turbulent plume. Conditions for the recovery of the Bragg scattering condition are established. (C) 1996 Acoustical Society of America.

PACS numbers: 43.30.Ft [JHM]

\section{INTRODUCTION}

Recently there has been considerable interest in applying acoustics to determine fluctuating oceanic fluid parameters, including changes in density, sound speed, and fluid velocity. In particular, the issue of remotely sensing fluid variability over volume reverberation due to biologics and particulates has been of increasing importance to the oceanographic, marine biology, and acoustics communities. During the past two decades there has been a limited amount of in situ measurements indicating that acoustic scattering from ocean microstructure is observable above volume reverberation. ${ }^{1-4}$ Limited accompanying environmental data for the in situ scattering experiments, however, restricted detailed knowledge of the state of the scattering volume in these experiments. Also, narrow bandwidth, monostatic acoustic systems limited the amount of information on the functional dependence of the scattering field. For an in-depth review of research relevant to the issue of acoustic scattering from ocean microstructure, see Goodman. ${ }^{5}$

In an attempt to describe the observed scattering strengths Goodman ${ }^{5}$ developed an empirically based model of ocean microstructure and, using weak scattering theory, predicted observed scattering strengths in terms of turbulence intensity levels and acoustic frequency. Predictions showed parameter regimes of acoustic scattering from microstructure to be on the order of that expected by particulates in the monostatic case. At near forward angles for the case of bistatic scattering, however, predictions indicated observable levels for scattering from microstructure above that from

a) Present address: Naval Research Laboratory, Code 7120, 4555 Overlook Ave. SW, Washington, DC 20375-5320. biologics and particulates. To date there exists no in situ bistatic scattering measurements to compare with these predictions.

Laboratory experiments on the scattering of sound from temperature, density, and fluid velocity fluctuations have been more widespread, but are still rather limited. Baerg and Schwarz ${ }^{6}$ performed a set of experiments of bistatic atmospheric acoustic scattering. Their work represents one of the most comprehensive data sets on the angular dependence of media variation on the scattering of sound from turbulence. A complete angular survey of the relative differential scattering cross section was made and compared with the theoretical predictions based on using a $-5 / 3$ power law spectrum for the turbulent field as set forth by Lighthill, ${ }^{7}$ Kraichnan, ${ }^{8}$ and Batchelor. ${ }^{9,10}$ The experimental results agreed with the theoretical calculations of the angular dependence of the relative scattering strength, showing enhanced scattering in the forward direction. Unfortunately, the system was not calibrated and no measurements of the absolute scattering strength were made.

In 1975, Brandt ${ }^{11}$ examined the issue of acoustic backscattering from density fluctuations produced by a saline jet. The mean density and fluctuation of the jet profile, and the size of the turbulent microscale along the jet centerline was calculated by using the results of conductivity measurements. The acoustic backscatter data was shown to correlate with the measured turbulent parameters.

Korman and Beyer ${ }^{12}$ reported on a laboratory experiment in which sound was scattered from a turbulent jet. Using the prescriptions of Kraichnan ${ }^{8}$ and Ishimaru, ${ }^{13}$ they measured the scattered spectra for near forward angles to 
determine the functional dependence of the spectral broadening and Doppler shift. Their results overestimated the variance of the turbulent velocity by $25 \%$, but their estimate of the mean jet velocity along the jet axis agreed well with the measured value.

Related laboratory experiments exist within the medical research community. ${ }^{14-22}$ Weak scattering theory is used to describe the scattering associated with ultrasonic imaging and tissue characterization. Tissue is well modeled as soft, fluid-like variations in compressibility and density satisfying the criterion for weak scattering theory. Unlike scattering from ocean microstructure where the dominant scattering mechanism is in most cases given by changes in compressibility, scattering from tissue includes contributions from density changes, as well as contributions from changes in compressibility. The central and common feature for the scattering process in both oceanic and medical applications is the scattering wave vector or what the authors have termed the Bragg wave vector. Although not the emphasis of most reports in the medical field, the Bragg wave vector, the variable at which the spatial Fourier transform of the scattering field is calculated, remains to be more fully utilized in both narrow and broad bandwidth applications.

For a discussion on the Bragg wave vector and the Bragg scattering condition, see Goodman et al. ${ }^{23}$ wherein they reported on results from a bistatic acoustic scattering experiment from a thermally produced laminar plume accompanied with a model based on far-field weak scattering theory. The experiment consisted of transmitting a narrow pulse, broad bandwidth signal, (240 kHz to $1.4 \mathrm{MHz})$ and measuring the scattered signal produced by the plume. The measurements were made from near forward to near backscatter scattering angles in a plane perpendicular to the plume axis. The scattering field was modeled as a cylindrically symmetric Gaussian with an $e$-folding value of $2.4 \mathrm{~mm}$ and an amplitude of $3.7^{\circ} \mathrm{C}$. This model resulted in an analytic expression for the scattered pressure field or, alternatively, the two-dimensional Fourier transform of the temperature field, $\phi$, and allowed direct comparison between the acoustic data and the predictions of weak scattering theory. Owing to the cylindrical symmetry of the laminar plume, the acoustic data could be inverted, providing an acoustic estimation of the temperature profile through the plume, which was then compared to the measured temperature profile. The results confirmed the prediction of the Bragg scattering condition, thus validating the usage of far-field weak scattering theory for the case of scattering from a laminar plume.

In the laminar plume experiment, the source was held in a fixed location while the receiver was moved to the various angles around a $25-\mathrm{cm}$-radius perimeter. Therefore, the magnitude and direction of the Bragg wave vector changed with each new scattering angle. For scattering in a plane of symmetry, variability along the Bragg wave vector is independent of direction. Consequently, for a fixed value of the Bragg wave number, $K$, the magnitude of the Fourier transform of the scattering field, $|\phi|$, is independent of the direction of the Bragg wave vector. Since the laminar plume is nearly cylindrically symmetric, $|\phi|$ is independent of direction. Data collected at different scattering angles from the laminar plume can then be compared on the same Bragg wave number axis. Broad bandwidth incident signals provide a corresponding range of Bragg wave numbers. The resulting domain of $\phi_{i}$ measured at scattering angle $\theta_{i}$ can then be directly compared to $\phi_{j}$ measured at $\theta_{j}$ whenever the measurements have equivalent Bragg wave numbers. The Bragg scattering condition can then be tested by comparing the agreement between the $\phi$ 's made at different scattering angles. For the special case of the laminar plume, the data confirmed the Bragg scattering condition.

When the condition of cylindrical symmetry is relaxed, the Fourier transform of the scattering field depends on both the magnitude and direction of the Bragg wave vector. However since the Bragg wave vector is given by $\mathbf{K}=\mathbf{k}_{s}-\mathbf{k}_{i}$, where $\mathbf{k}_{s}$ and $\mathbf{k}_{i}$ are the scattered and incident wave vectors, respectively, Bragg wave vectors will be coincident in direction whenever source-receiver pairs share a common bisector. By using two different transmit frequencies such that $K_{1}=2 k_{1} \sin \left(\theta_{1} / 2\right)=2 k_{2} \sin \left(\theta_{2} / 2\right)=K_{2}$, scattering can result at exactly the same Bragg wave number. The Bragg scattering condition predicts identical $|\phi|$ 's for equal Bragg wave numbers. By combining broad bandwidth transmissions/receptions with multiple simultaneous scattering measurements in the same scattering direction a more fully resolved Fourier transform of the scattering field can be obtained. This type of experiment is termed the common scattering direction experiment and will be discussed in a subsequent manuscript. This work will concentrate on the case of scattering with Bragg wave vectors identical in magnitude, but oppositely directed.

The far-field Born approximation predicts equal magnitudes for the Fourier transforms of the scattering field for this case. It is shown that scattering from spatial variability of order the Fresnel radius, $z_{F}=\sqrt{r_{0} \lambda}$, results in wavefront curvature effects being important. This, theoretically, invalidates the Bragg scattering condition and results in different scattering transfer functions from oppositely directly Bragg wave vectors of the same magnitude. This case is examined in the laboratory. In the next section we will examine the theoretical relationships expected for scattering from a thin buoyant plume.

\section{THEORY OF ACOUSTIC SCATTERING FROM A THIN THERMALLY DRIVEN BUOYANT PLUME}

Scattering occurs when sound travels into a region characterized by a change in either density, compressibility, or the fluid velocity. The equation of motion is given by ${ }^{24,25}$

$$
\left(\nabla^{2}-\frac{1}{c^{2}} \frac{\partial^{2}}{\partial t^{2}}\right) P(\mathbf{r}, t)=L(\mathbf{r}, t) P(\mathbf{r}, t),
$$

where $P(\mathbf{r}, t)$ is the pressure at position $\mathbf{r}$ and time $t$ and $L$, the scattering operator is given by

$$
\begin{aligned}
L(\mathbf{r}, t)= & \frac{1}{c^{2}} \gamma_{\kappa}(\mathbf{r}, t) \frac{\partial^{2}}{\partial t^{2}}+\boldsymbol{\nabla} \cdot\left(\gamma_{\rho}(\mathbf{r}, t) \boldsymbol{\nabla}\right)+\frac{1}{c^{2}} \frac{\partial \mathbf{u}}{\partial t} \cdot \boldsymbol{\nabla} \\
& +\frac{2}{c^{2}} \mathbf{u} \cdot \boldsymbol{\nabla} \frac{\partial}{\partial t},
\end{aligned}
$$


where $c_{0}=1 / \sqrt{\kappa_{0} \rho_{0}}$ is the ambient sound speed, $\kappa_{0}$ is the ambient compressibility, $\rho_{0}$ is the ambient density, $\gamma_{\kappa}=\left(\kappa-\kappa_{0}\right) / \kappa_{0}$ is the relative compressibility, $\gamma_{\rho}=\left(\rho-\rho_{0}\right) / \rho$ is the relative density, and $\mathbf{u}$ is the fluctuation of the fluid velocity.

For the thermally produced buoyant plume considered in this work, the dominant scattering mechanism is variation of the relative compressibility from temperature fluctuations produced by a buoyant thermally driven plume. (This type of scattering was examined by the authors in a previous manuscript. ${ }^{23}$ ) As a result, only the first term in Eq. (2) is significant $^{5,23}$ and $\gamma_{\kappa}=-2 \alpha T$, where $\alpha=\left(1 / c_{0}\right)(d c / d T), T$ the temperature difference from ambient.

If we define the Fourier transform for the pressure field as

$$
p(\mathbf{r}, \omega)=\int P(\mathbf{r}, t) \exp (i \omega t) d t
$$

then $L=\left(-2 \alpha T / c^{2}\right)\left(\partial^{2} / \partial t^{2}\right)$ and Eq. (1) in frequency space is given by

$$
\left(\nabla^{2}+k^{2}\right) p(\mathbf{r}, \omega)=-k^{2} \gamma_{\kappa}(\mathbf{r}) p(\mathbf{r}, \omega),
$$

where $k=\omega / c$.

The Born approximation solution for the scattered pressure field in Eq. (4) for the boundary-free case is given by

$$
p=-2 \alpha k^{2} \int T\left(\mathbf{r}^{\prime}\right) B\left(\mathbf{r}^{\prime}\right) p_{0}\left(\mathbf{r}^{\prime}, \omega\right) g\left(\mathbf{r} \mid \mathbf{r}^{\prime}\right) d \mathbf{r}^{\prime},
$$

where

$$
g\left(\mathbf{r} \mid \mathbf{r}^{\prime}\right)=\frac{\exp \left(i k_{s}\left|\mathbf{r}-\mathbf{r}^{\prime}\right|\right)}{4 \pi\left|\mathbf{r}-\mathbf{r}^{\prime}\right|} \approx \frac{\exp \left(i k\left|\mathbf{r}-\mathbf{r}^{\prime}\right|\right)}{4 \pi r}
$$

is the point source Green's function. The weighting function $B\left(\mathbf{r}^{\prime}\right)$ is the source/receiver beam pattern; the incident pressure is given by $p_{0}=P_{0} \exp \left(i k r^{\prime}\right)$. The radial dependence of the incident field is absorbed in the definition of $P_{0}$. The Born approximation is expected to be valid when ${ }^{26}$

$$
\alpha T^{*} k L^{*} \ll 1
$$

where $L^{*}$ is the characteristic value of the length scale of the scattering field in the plane of the Bragg wave number vector, and $T^{*}$ the value of the temperature fluctuation of the thermally driven buoyant plume.

In Fig. 1 the geometry of the scattering process is shown. The scattering volume has linear dimensions $L^{*} \ll r_{0}$, where $r_{0}$ is the distance from source and receiver to the center of the scattering volume, taken as equidistant, the experimental case discussed in Sec. II. It is convenient to define the spatial coordinate system, as shown in Fig. 1, aligned with the " $x$ " axis along the Bragg wave vector defined by $\mathbf{K}=\mathbf{k}_{s}-\mathbf{k}_{i}$, where $\mathbf{k}_{i}, \mathbf{k}_{s}$ are the incident and scattered wavenumber vectors whose magnitude is $k$ and whose directions are, respectively, the incident center line direction of lines SO and OR of Fig. 1. The magnitude of $\mathbf{K}$ is given by $|\mathbf{K}|$ $=2 k \sin (\theta / 2)$ with its direction along the bisector of SOR, again taken along the " $x$ " axis. Defining $\Gamma$ as the transfer function of the scattering process and expanding the phase term of Eq. (5) $\left|\mathbf{r}-\mathbf{r}^{\prime}\right|$ to lowest order results in

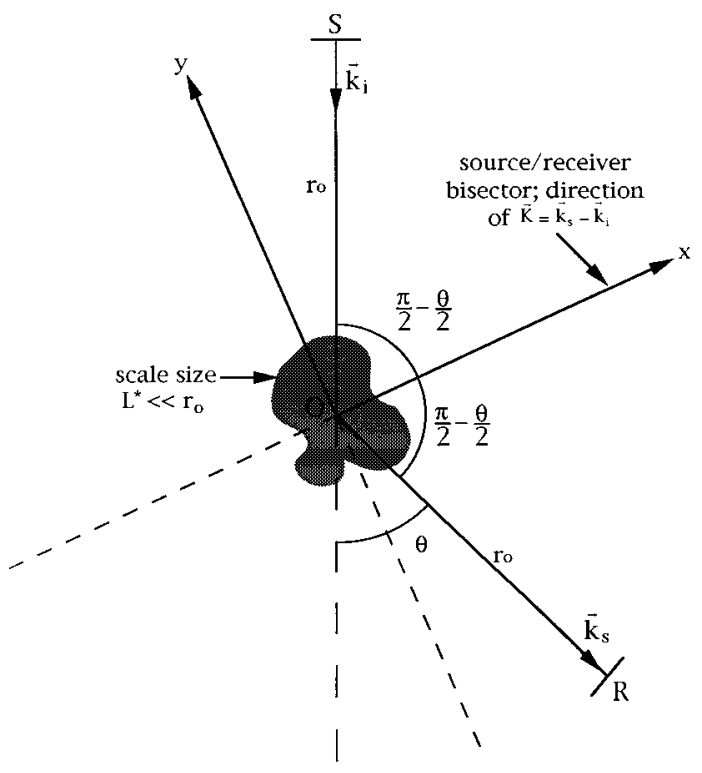

FIG. 1. Scattering geometry for experiment. source, $S$, and receiver, $R$, are in $x-y$ plane.

$$
\Gamma(K) \equiv \frac{p}{p_{0}}=\frac{\alpha k^{2}}{2 \pi r_{0}} \int T(\mathbf{x}) B(\mathbf{x}) \exp [i K x+i k \zeta] d^{3} x,
$$

where

$$
\zeta=z^{2} / r_{0}
$$

is the Fresnel curvature term. It is straightforward to show for the experimental results presented below that terms higher order than this term make insignificant contributions to the phase, i.e., $k$ times (higher-order terms) $\ll 1$. Also because of the thinness of the buoyant plume relative to the beam pattern only the " $z$ ", dependence in $B$ needs to be considered. These two sets of assumptions-dropping the higher-order phase terms and only considering the $z$ dependence of $B$-are discussed in the Appendix. Note that when $k \zeta \ll 1$ the phase correction terms in Eq. (8) can be ignored and

$$
\Gamma(K)=\frac{\alpha k^{2}}{2 \pi r_{0}} \int T(\mathbf{x}) B(z) \exp [i K x] d^{3} x \equiv \frac{\alpha k^{2}}{2 \pi r_{0}} \phi(K),
$$

where $\phi$ is the beam pattern weighted or filtered (in the " $z$," direction) Fourier transform of the temperature field at the Bragg wave number $K$. Equation (10) allows a straightforward inversion to obtain the temperature field from the scattered acoustic pressure field, by inferring from the acoustic measurements of $\Gamma$

$$
\phi(K)=\frac{2 \pi r_{0} \Gamma(K)}{\alpha k^{2}}
$$

over all magnitudes and directions of $\mathbf{K}$ and then inverse transforming to obtain $T(\mathbf{x})$. Unfortunately because real acoustic systems have finite bandwidth and because multidirectional (including in different planes) measurements have practical limitations, use of (11) is limited. Nonetheless Eq. (11) is a clear prescription for inferring important aspects of 


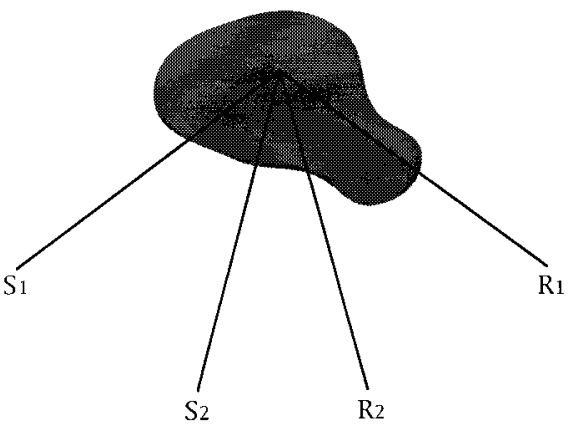

FIG. 2. Common Bragg wave-number direction geometry.

the temperature field of a fluid. Clever usage of Bragg scattering geometry can also be made to enhance the "effective", bandwidth over which $\phi$ is being inferred. For the experiments sited below the bandwidth of the system is between 250 and $750 \mathrm{kHz}$ (10-dB power down points). By performing a series of simultaneous multiple pair scattering angle experiments such that the Bragg wave number lies in the same direction, which is accomplished by having each source/receiver transducer pair have a common bisector, the " $x$ ', axis (Fig. 2) but different scattering angles, $\theta_{1}, \theta_{2}, \theta_{3}$, etc., the effective spatial bandwidth in $\phi$ can be expanded from a factor of 3 to about a factor of 34 for the case of $\theta=10^{\circ}$ to $\theta=180^{\circ}$ as shown in Fig. 3. However if the phase term, Eq. (9), is significant in Eq. (8) the Fourier transform $\phi$ does not separate out of the integral of Eq. (8). Nevertheless it is straightforward to show that Eq. (8) can be rewritten in terms of both the " $x$ '" and " $z$ " wave-number transform variables on $\phi$ as

$$
\Gamma(K, \tau)=\frac{\alpha k^{2}}{2 \pi r_{0}} \int \phi\left(K, k_{z}, \tau\right) g\left(k, k_{z}\right) d k_{z},
$$

where

$$
\phi\left(K, k_{z}, \tau\right)=\int T(\mathbf{x}, \tau) \exp \left[i K x+i k_{z} z\right] d^{3} x
$$

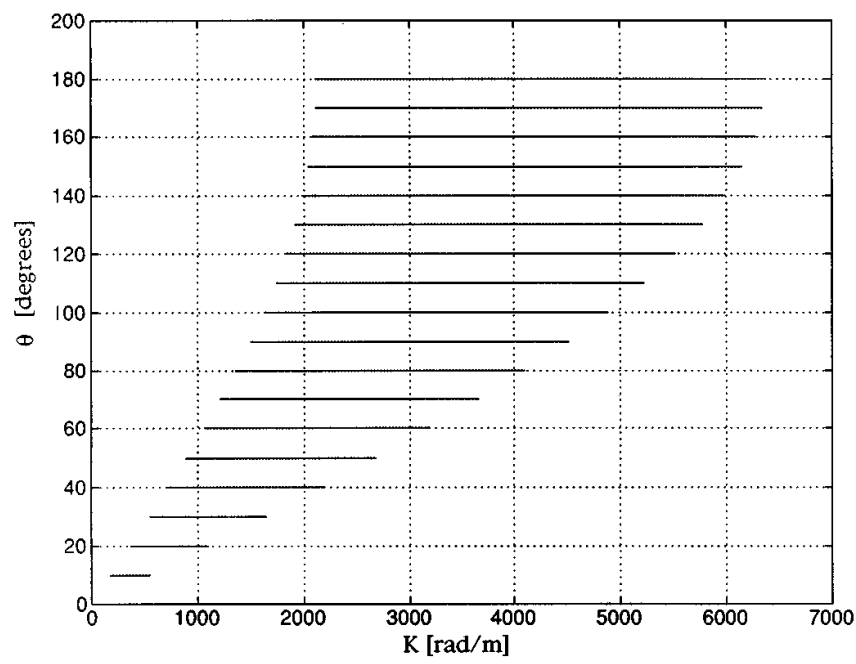

FIG. 3. Bragg wave-number bandwidth $\Delta K$ for $250 \mathrm{kHz}<f<750 \mathrm{kHz}$, $\Delta K=2(2 \pi \Delta f / c) \sin (\theta / 2)$, where $\Delta f=500 \mathrm{kHz}$. $g$ is a filter function arising from wavefront curvature and beam pattern amplitude weighting, namely,

$$
g\left(k, k_{z}\right)=\int B(z) \exp \left[i k_{z} z+\frac{i k z^{2}}{r_{0}}\right] d z .
$$

Note in Eq. (12) we have suppressed the wave-number dependence $k$ and explicitly included time dependence in the notation for $\Gamma$ since in the experiments to be discussed the time dependence is an important factor to be exploited. Equations (12) and (14) show that the beam pattern amplitude weighting, $B(z)$, and the phase effects of wavefront curvature, $i k z^{2} / r_{0}$, both result in filtering of the temperature field over some finite bandwidth in " $k_{z}$ " space. Equation (10) assumes such filtering removes all wave numbers except near $k_{z} \approx 0$. For this case neglecting the phase term $i k z^{2} / r_{0}$, and setting $B(z)=1$, Eq. (14) becomes $g\left(k, k_{z}\right)=2 \pi \delta\left(k_{z}\right)$ and Eq. (10) follows from (12). Note that estimating $z_{b}=r_{0} / k a$ for the vertical extent of the half-beamwidth in the " $z$ " direction, where " $a$ " is a cylindrical transducer radius, and $z_{F}=\sqrt{2 \pi r_{0} / k}$ the vertical extent of the Fresnel radius, in the far field by definition ${ }^{27}\left(z_{F} / z_{b}\right)^{2} \ll 1$ and Eq. (14) can be approximated by

$$
g\left(k, k_{z}\right)=\sqrt{\frac{i}{2}} z_{F} \exp \left[-i \frac{\left(k_{z} z_{F}\right)^{2}}{8 \pi}\right] .
$$

Equations (12) and (14) or Eq. (12) with the "far-field", approximation (15) show that when wavefront curvature effects are important that in general

$$
\Gamma(K, \tau) \neq \Gamma^{*}(-K, \tau)
$$

but if wavefront curvature is unimportant, i.e., $k \zeta \ll 1$, that

$$
\Gamma(K, \tau)=\Gamma^{*}(-K, \tau) .
$$

Conditions under which either Eqs. (16) or (17) hold can be examined in the laboratory by performing a scattering experiment involving two pairs of sources/receivers oriented such that their Bragg wave numbers are of the same magnitude but opposite direction. Let $\Gamma_{+}$and $\Gamma_{-}$refer to the transfer functions obtained from the respective positive and negative Bragg wave vectors of such an experiment, where $\Gamma$ is obtained from its definition in Eq. (8). In general Eq. (16) holds, however there are circumstances in which although wavefront curvature effects are significant, $\Gamma_{+}$and $\Gamma_{-}$have a simple relationship. Two types of assumptions on the nature of the variability of the fluid field allow such relationships to emerge. These are (1) the plume motion satisfies Taylor's hypothesis in the " $z$ "' direction; and (2) the plume temperature variability is statistically homogeneous and stationary. The former will be applied to an unstable, nonturbulent plume, while the latter to a turbulent plume. We will complete this section by examining the consequences of these assumptions and criteria of their application.

\section{A. Application of Taylor's hypothesis to plume variability}

For the case of using Taylor's hypothesis it is assumed that the temperature field of the buoyant plume satisfies

$$
T(x, y, z, \tau)=T\left(x, y, z-z_{0}\right),
$$




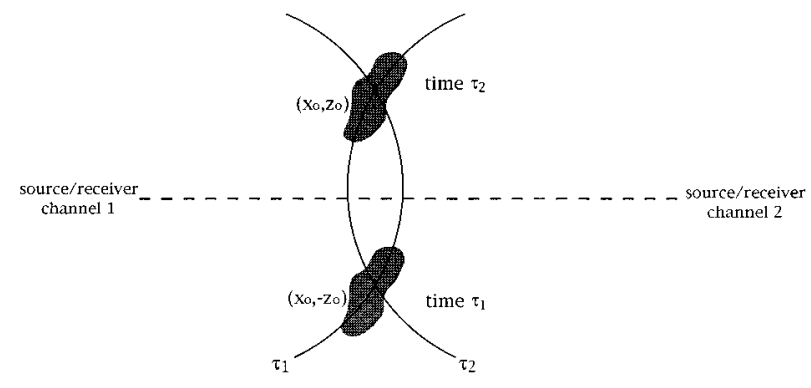

FIG. 4. Opposing Bragg wavefronts for a monostatic case illustrating application of Taylor's hypothesis.

where $z_{0}=w \tau, w$ is the vertical advection velocity, and $\tau$ is the time associated with the repetition rate of the experiment. This is valid when the variability of the fluid field is such that $^{28}$

$$
t^{*} \gg L^{*} / w,
$$

where $t^{*}$ and $L^{*}$ are the characteristic time and length scale of a plume eddy being advected in the vertical with speed $w$. Use of Taylor's hypothesis in Eq. (13), is equivalent to performing a set of simultaneous scattering experiments in a series of planes $z_{0}$. The Fourier transform of (12) on $z_{0}=w \tau$ can be written

$$
\beta=\int \Gamma \exp \left(i k_{z} z_{0}\right) d z_{0}=\frac{\alpha k^{2}}{2 \pi r_{0}} g\left(k, k_{z}\right) \phi\left(K, k_{z}\right),
$$

where in Eq. (13) we have used Taylor's hypothesis $T(\mathbf{x}, \tau)$ $=T(\mathbf{x}-\mathbf{w} \tau)$ to obtain the relationship $\phi\left(K, k_{z}, \tau\right)$ $=\phi\left(K, k_{z}\right) \exp \left[i k_{z} w \tau\right]$.

From (20) the inversion

$$
\phi\left(K, k_{z}\right)=\frac{2 \pi r_{0} \beta}{\alpha k^{2} g\left(k, k_{z}\right)}
$$

follows, and that

$$
\phi_{ \pm}\left(K, k_{z}\right)=\frac{2 \pi r_{0} \beta_{ \pm}}{\alpha k^{2} g\left(k, k_{z}\right)},
$$

where $\beta_{ \pm}$defined by Eq. (20) is the Fourier transform in $z_{0}$ of $\Gamma_{ \pm}$. From (21) we see that Taylor's hypothesis allows calculation of the wave-number transform in $K, k_{z}$ space rather than just $K$ space since the advection in the vertical brings past the vertical scattering plane information. Equation (22) establishes a testable relationship between $\beta_{ \pm}$, namely, that

$$
\frac{\beta_{+}\left(K, k_{z}\right)}{g\left(k, k_{z}\right)}=\frac{\beta_{-}\left(-K, k_{z}\right)}{g\left(-k, k_{z}\right)}
$$

and that since $g\left(-k, k_{z}\right)=g^{*}\left(k, k_{z}\right)$ that

$$
\left|\beta_{+}\left(K, k_{z}\right)\right|=\left|\beta_{-}\left(-K, k_{z}\right)\right| .
$$

In Fig. 4 the physics underlying the symmetry imposed by the role of Taylor's hypothesis in relating $\beta_{ \pm}$is illustrated. Consider a scatterer (a fluid parcel of some temperature anomaly) at time $\tau_{1}$ located at $\left(x_{0},-z_{0}\right)$ lying along the lower part of the convex wavefront emitted from source/ receiver pair 1 . Taylor's hypothesis implies that this same scatterer will be located at $\left(x_{0}, z_{0}\right)$ at some latter time, $\tau_{2}$,

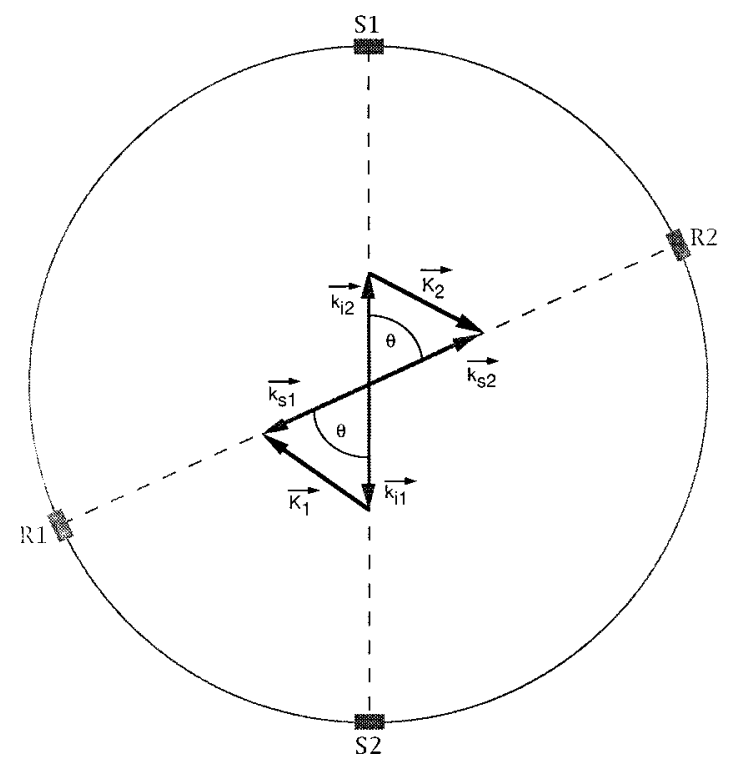

FIG. 5. Opposing Bragg wave-number vector geometry.

and thus will be located at exactly the mirror reversed position of the oppositely directed wavefront from source/ receiver 2. Both wavefronts receive the same magnitude and phase but time delayed $\tau_{2}-\tau_{1}$. In reality the fluid will change its properties (magnitude and orientation of its temperature anomaly field) as it traverses from $\left(x_{0},-z_{0}\right)$ to $\left(x_{0}, z_{0}\right)$ but if (18) is satisfied, this change is sufficiently small that it can be neglected. Since the wavefront acts as a filter over scales of order the Fresnel radius $z_{F}=\sqrt{r_{0} \lambda}$, the time scales of variability should satisfy

$$
t^{*} \gg \frac{z_{F}}{w}
$$

\section{B. Application of homogeneity and stationarity to plume variability}

To form a wave-number spectrum it is necessary to assume, at least locally, a random field satisfies homogeneity and stationarity. ${ }^{10}$ From (13) this condition can be written

$$
\left\langle\phi\left(K, k_{z}, \tau\right) \phi^{*}\left(K, k_{z}^{\prime}, \tau\right)\right\rangle=\Phi\left(K, k_{z}\right) \delta\left(k_{z}-k_{z}^{\prime}\right)
$$

for which from Eq. (12) it follows that the wave-number spectrum is given by

$$
\begin{aligned}
\Psi & \equiv\left\langle\Gamma(K, \tau) \Gamma^{*}(K, \tau)\right\rangle \\
& =\left(\frac{\alpha k^{2}}{2 \pi r_{0}}\right)^{2} \int d k_{z} \Phi\left(K, k_{z}\right)\left|g\left(k, k_{z}\right)\right|^{2} .
\end{aligned}
$$

Thus $\Psi(K)\left(\alpha k^{2} / 2 \pi r_{0}\right)^{-2}$ is the wave-number spectrum of the temperature field filtered in the $k_{z}$ by $|g|^{2}$. Note that (25) predicts the same spectrum for $\Psi_{ \pm}$, since $\Psi(K)=\Psi(-K)$. The validity of Eq. (25) will be examined in laboratory experiments.

\section{EXPERIMENTAL SETUP}

The principle objective of the experiment is to examine the validity of Eq. (16) or Eq. (17). Equation (16) requires 


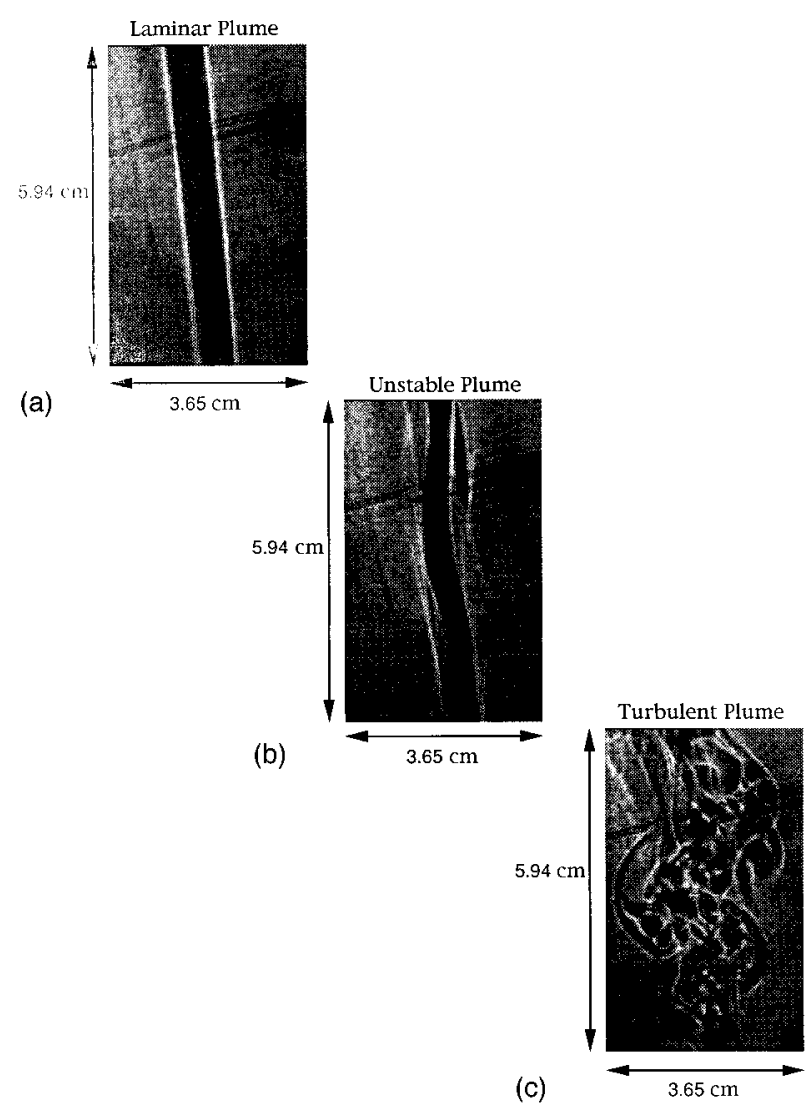

FIG. 6. Laser shadowgraph images for (a) laminar plume, (b) unstable plume, and (c) turbulent plume.

that wavefront curvature effects be accounted for in order to interpret the scattering results in terms of the temperature field. Note that Eq. (17) predicts that for oppositely directed incident wave fields with the same magnitude Bragg wavenumber vector, the geometry of which is shown in Fig. 5, the same magnitude of $\Gamma$ results, i.e., $\left|\Gamma_{+}\right|=\left|\Gamma_{-}\right|$. For the case of an axisymmetric laminar plume experimental results have been consistent with this relationship. ${ }^{23}$ This arose, however, because of the cylindrical symmetry of the laminar plume, i.e., $\phi\left(-k, k_{z}\right)=\phi\left(k, k_{z}\right)$, the steady (unchanging in time) nature of the variability field, and local uniformity in the vertical direction. This experiment will examine whether the Bragg scattering condition on the laminar plume can be extended to the cases of scattering from an unstable and turbulent plume, where no such symmetry, in general occurs, and which is intrinsically unsteady in time. Because of the threedimensional variation of the temperature field for the unstable and turbulent cases unlike the laminar plume case ${ }^{23}$ it is not possible to do a direct comparison between the temperature field (or its Fourier transform) observed acoustically and that inferred from mechanical sensors (thermistors), since such sensors are point measurements and the acoustic inversion [Eq. (11)] involves a spatially integrated measurement. Instead the point of view adopted here is, given the previous positive results on the laminar plume case where a direct comparison was in good agreement, to see whether the data obtained is consistent with the constraint imposed by Eq. (17) and, if not, with the hypotheses presented at the end of Sec. I.
In Fig. 6(a)-(c) the laser shadowgraph images are shown for the three plume fluid stability regimes. The laser shadowgraph is used during the experiments as a visual monitor. The scattering experimental setup consists of a Plexiglas tank filled with filtered fresh tap water. Inside the tank is placed a ring assembly system consisting of two plates. On the bottom plate is a $3 / 8$-in.-diam, $50-\mathrm{W}$ heating element used to generate a buoyant plume. The top plate is a $50-\mathrm{cm}$ i.d. ring, around which transducers can be placed in 10-deg increments. For this experiment, the transducers are arranged as shown in Fig. 5. The scattering angles for source/ receiver pairs $S_{1}, R_{1}$ and $S_{2}, R_{2}$ are chosen as $30 \mathrm{deg}$ to produce the maximum signal with minimum sidelobe effects and a spatial wave-number bandwidth sufficiently wide to resolve the plume temperature variability. By varying the input power to the heating element, three different parameter regimes can be examined: laminar, unstable, and turbulent.

Three types of acoustic measurements are made in order to calculate $\Gamma$, defined by Eq. (8).

(1) To calculate the incident pressure field, a direct path measurement is made between each source and receiver with the receiver placed at the center of the scattering volume.

(2) A "plume on" measurement in which the scattered signal from the plume is range gated and digitized.

(3) A "plume off" measurement which is used to subtract from the scattered signal any stationary reverberation, i.e., $\quad P_{s}(x, \tau)=P_{\text {on }}(x, \tau)-\bar{P}_{\text {off }}(x), \quad$ where $\quad \bar{P}_{\text {off }}(x)$ $=\left\langle P_{\text {off }}(x, \tau)\right\rangle$. The transmit signal consists of single-cycle waveform with center frequency of $500 \mathrm{kHz}$, amplified by a $2-\mathrm{kW}$ power amplifier. The transducers have a 10-dB down point bandwidth of $500 \mathrm{kHz}$, i.e., 250 to $750 \mathrm{kHz}$. At time $\tau=0$ source 1 transmits the single-cycle signal, receiver 1 is range gated at the plume and the received signal is digitized at $5 \mathrm{MHz}$. The waveforms are stored in place onboard the data acquisition system and off loaded after the completion of the experiment. Since the scattering field is, in general, time dependent, it is necessary to measure the scattering for source/receiver pair 2 as near simultaneous as possible with source receiver/pair 1. Typically, reverberation persists on the order of milliseconds, while the time variability of the plume is on the order of hundreds of milliseconds and longer. So that a transmit time for source 2 at $\tau=5 \mathrm{~ms}$ after that of source 1 is sufficiently small to ensure that source/ receiver pair 2 interrogates the same scattering field as source/receiver pair 1 . The system repetition interval is chosen as $15 \mathrm{~ms}$. This cycle is repeated 1023 times for a total of approximately $15 \mathrm{~s}$ of plume variability data. Results from two cases will be presented below: Case A, the nonturbulent but unstable plume Fig. 6(b), and case B the turbulent plume Fig. 6(c).

\section{EXPERIMENTAL RESULTS}

\section{A. Case A: Unstable nonturbulent plume}

The terms channels 1 and 2 refer to scattering associated with source/receiver pairs $S_{1}, R_{1}$ and $S_{2}, R_{2}$, respectively, corresponding to opposite Bragg wave-number directions and thus $\pm K$. Contour plots of the raw magnitude of the scattered signals, unnormalized pressure amplitude, $|P|$, for 


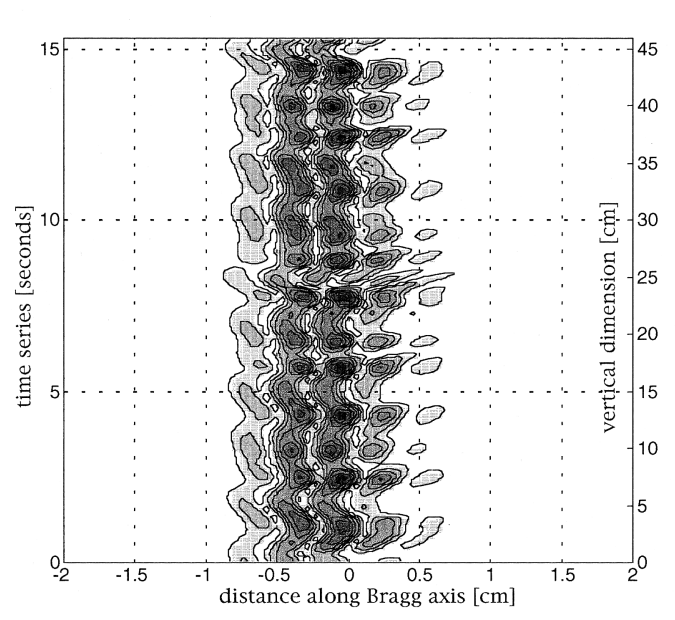

FIG. 7. Contour plot of the magnitude of the scattered signal from an unstable plume for channel 1.

channels 1 and 2 are displayed in Figs. 7 and 8. The left hand ordinate represents the time series in units of seconds which is formed from returns at the system repetition rate of $15 \mathrm{~ms}$. The right hand ordinate has been converted to a spatial coordinate by use of a mean vertical plume velocity of $3 \mathrm{~cm} / \mathrm{s}$ inferred from laser shadowgraph tracking of features. The abscissa is along the vector Bragg wave-number direction, i.e., " $x$ " axis in Fig. 1 in units of $\mathrm{cm}$, defined in terms of channel 1 source/receiver location. In Fig. 8 the " $x$ " axis coordinate used is the same as in Fig. 7 and so positive values actually correspond to earlier arrivals.

Note that the two figures are qualitatively similar but have a mirror image type of asymmetry. Both have approximately the same number of local maxima with a periodicity of about $1.5 \mathrm{~s}$ corresponding to $5 \mathrm{~cm}$. An anomalous feature is noted in both figures at about $8 \mathrm{~s}$. The strong steady periodicity noted in these features is expected from the unstable nonturbulent plume shown in Fig. 6(b) which does have about the same vertical spatial scale as the acoustic measurements. (However it should be noted that the aperture of the laser was limited to $6 \mathrm{~cm}$ in the vertical.) There does appear to be a phase lag between the maxima of channels 1 and 2 of
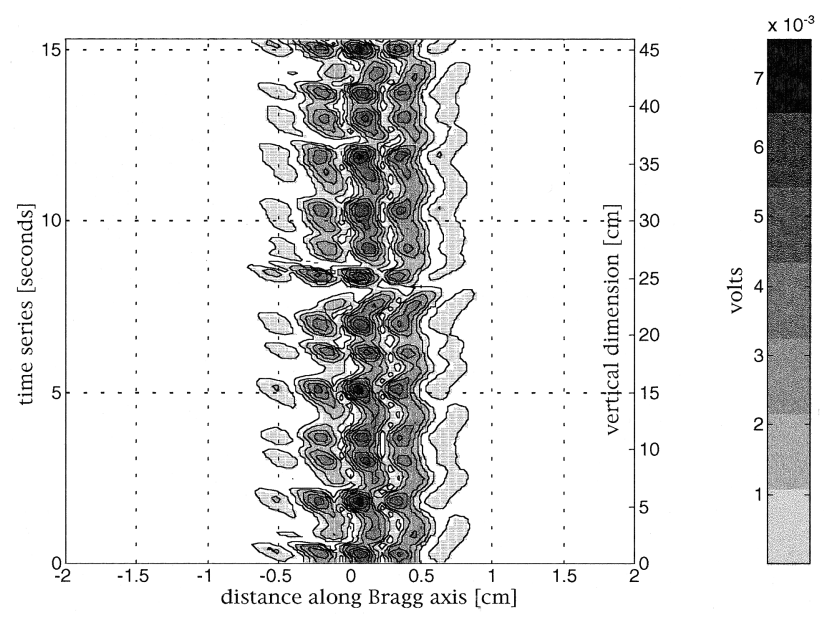

FIG. 8. Contour plot of the magnitude of the scattered signal from an unstable plume for channel 2.

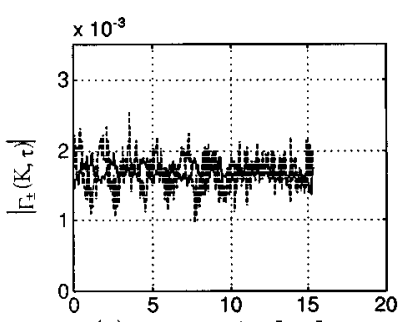

(a) time series [sec]

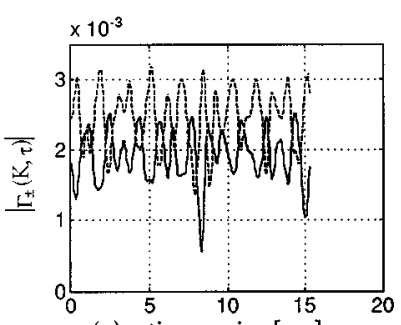

(c) time series [sec]

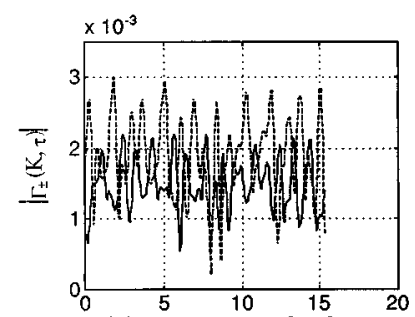

(e) time series [sec]

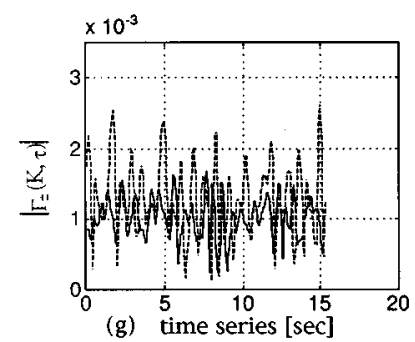

(g) time series $[\mathrm{sec}]$

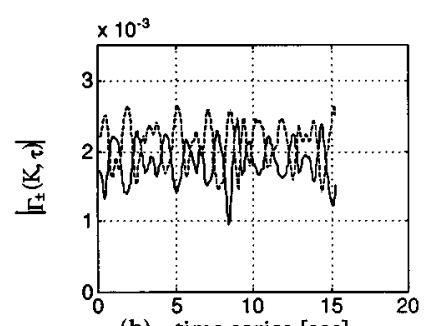

(b) time series [sec]

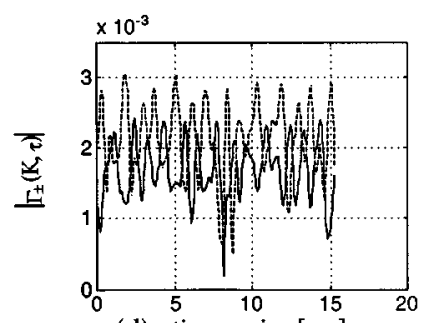

(d) time series [sec]

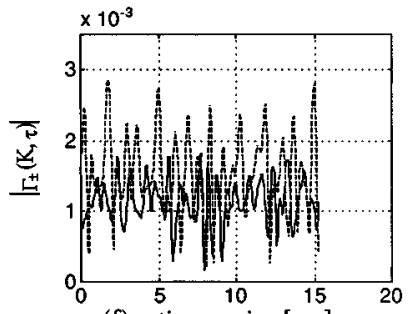

(f) time series [sec]

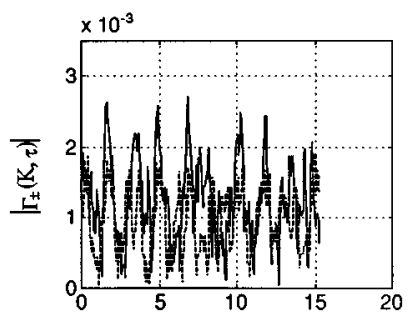

(h) time series $[\mathrm{sec}]$
FIG. 9. Time series of $\left|\Gamma_{ \pm}(K, \tau)\right|$ for frequencies of (a) $117 \mathrm{kHz}$, (b) 234 $\mathrm{kHz}$, (c) $313 \mathrm{kHz}$, and (d) $430 \mathrm{kHz} . \Gamma_{+}, \Gamma_{-}$is given by solid/dashed lines, respectively. Time series of $\left|\Gamma_{ \pm}(K, \tau)\right|$ for frequencies of (e) $508 \mathrm{kHz}$, (f) $625 \mathrm{kHz},(\mathrm{g}) 703 \mathrm{kHz}$, and (h) $938 \mathrm{kHz} . \Gamma_{+}, \Gamma_{-}$is given by solid/dashed lines, respectively.

about $1 \mathrm{~s}$ or about $3 \mathrm{~cm}$. We attribute this phase lag to the effect described in Fig. 4 where a feature which lies along the slope of the wavefront from one of the channels will be advected vertically and at some latter time lie along the same sign slope of the wavefront of the other channel. It is interesting to note that $3 \mathrm{~cm}$ does correspond to order of the Fresnel radius at the center frequency $500 \mathrm{kHz}$.

It should be noted that Figs. 7 and 8 contain effects due to differences in the individual transducer characteristics and thus should be viewed as qualitative indicators of the scattering. These effects are removed in the calculation of $\Gamma$ versus time, which is displayed in Fig. 9(a)-(h) for the same data set of Figs. 7 and 8. The range of Bragg wave numbers presented correspond to the bandwidth of the system. Figure 9(a) is at frequency well below the response range of the transducer and is an indicator of noise. At Bragg wave numbers less than $1116 \mathrm{rad} / \mathrm{m}(f=508 \mathrm{kHz})$ the mean value of $|\Gamma|$ of the two curves are different. However the variance of fluctuations are of similar magnitude. At Bragg wave num- 


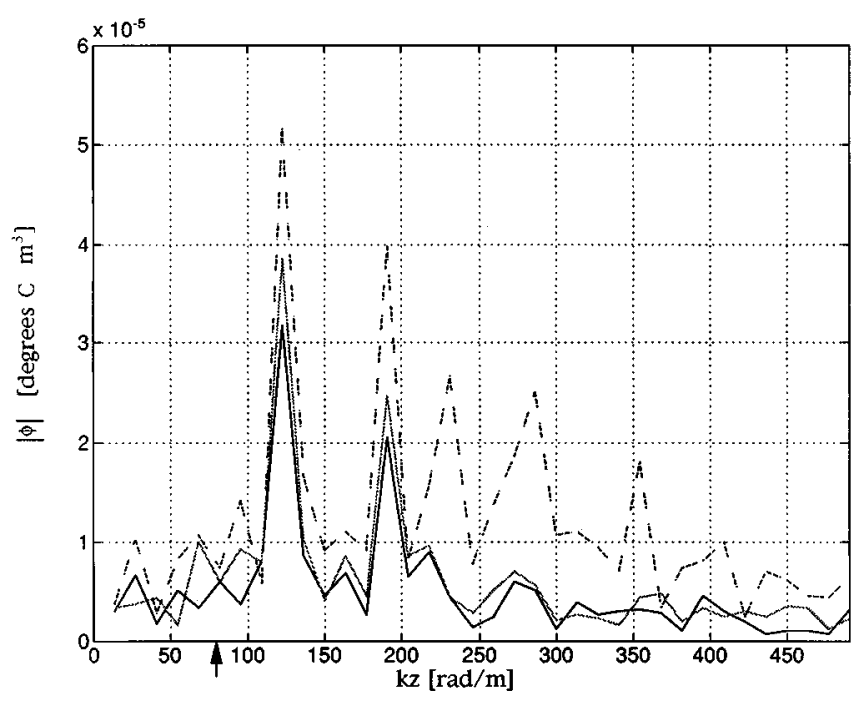

FIG. 10. Slice of the two component three-dimensional Fourier transform of temperature field, $|\phi|$, at $K=515 \mathrm{rad} / \mathrm{m}(f=234 \mathrm{kHz})$. Solid black/gray lines indicate $\left|\phi_{+}\right|$and $\left|\phi_{-}\right|$, respectively, dashed black line is $\left|\phi_{+}\right|$at $K=-515$ $\mathrm{rad} / \mathrm{m}$.

bers greater than $1116 \mathrm{rad} / \mathrm{m}$ the mean value of $|\Gamma|$ of the two curves converge but their variances are different with channel 2 having consistently larger variance.

The fact that there appears to be a phase lag between features in Figs. 7 and 8 suggests the possible use of Taylor's hypothesis for this data set. Equation (21) can then be used to estimate the wave-number transform $\phi\left(K, k_{z}\right)$ from the Fourier transform of $\Gamma$ on $z_{0}=$ wt. Using $w=3 \mathrm{~cm} / \mathrm{s}$, estimated from the laser shadowgraph data, in Eq. (20) with $\beta_{ \pm}$ calculated from $\Gamma_{ \pm}$associated with the two channels estimated values of $\left|\phi_{ \pm}\left(K, k_{z}\right)\right|$ are plotted versus $k_{z}$ in Figs. 10 and 11 for Bragg wave numbers of $K=515 \mathrm{rad} / \mathrm{m}(f=237$ $\mathrm{kHz})$ and $K=1373 \mathrm{rad} / \mathrm{m}(f=633 \mathrm{kHz})$. The plus/minus subscript refer to estimations from data obtained from channels one and two, respectively. Note the strong peaks at $k_{z}=120$

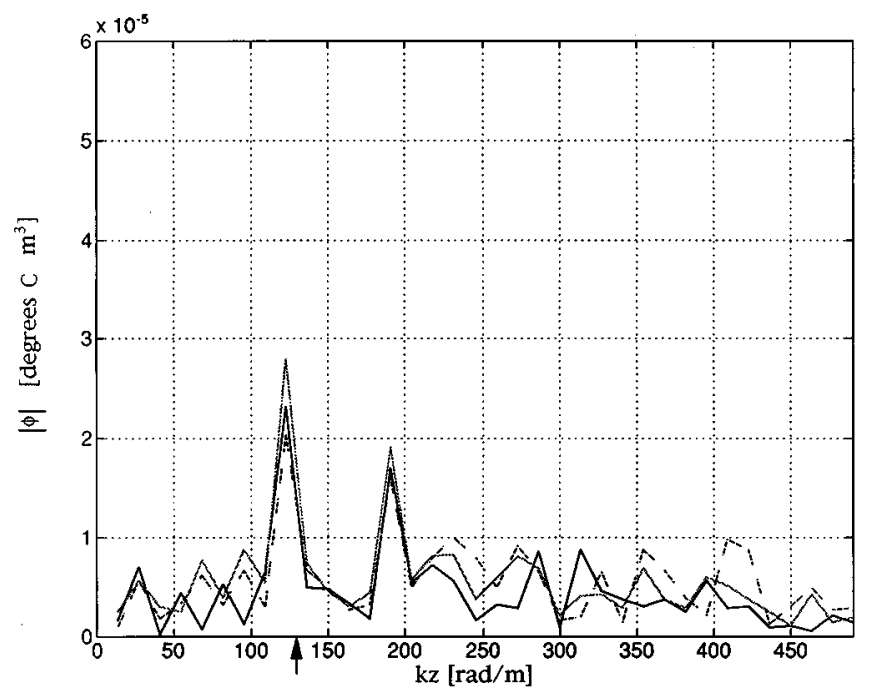

FIG. 11. Slice of the two component three-dimensional Fourier transform of temperature field, $|\phi|$, at $K=1373 \mathrm{rad} / \mathrm{m}(f=625 \mathrm{kHz})$. Solid black/gray lines indicate $\left|\phi_{+}\right|$and $\left|\phi_{-}\right|$, respectively, dashed black line is $\left|\phi_{+}\right|$at $K=-1373 \mathrm{rad} / \mathrm{m}$.

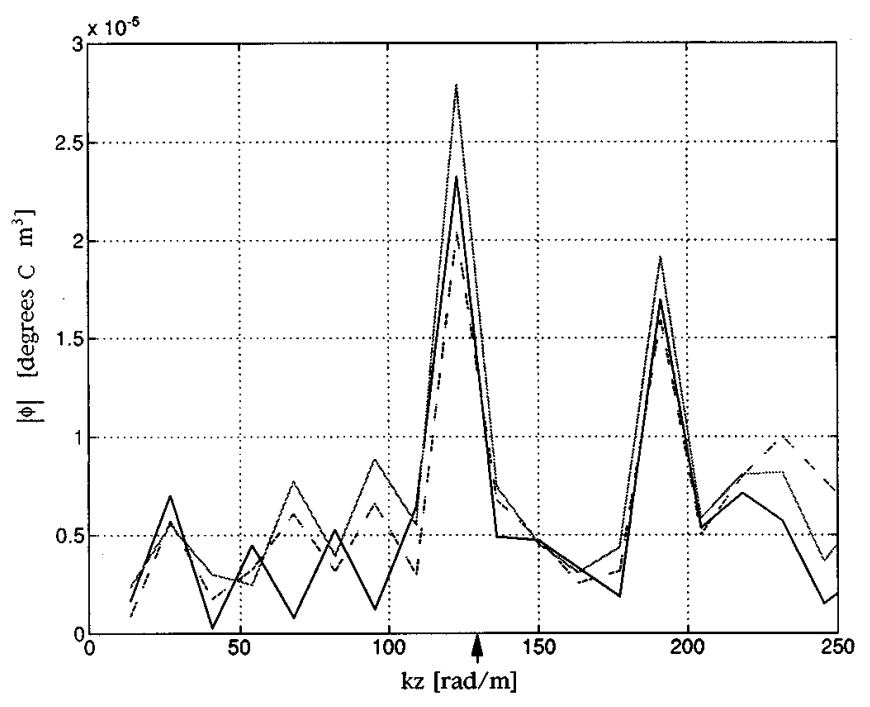

FIG. 12. Expanded graph of the two component three-dimensional Fourier transform of temperature field, $|\phi|$, at $K=1373 \mathrm{rad} / \mathrm{m}(f=625 \mathrm{kHz})$. Solid black/gray lines indicate $\left|\phi_{+}\right|$and $\left|\phi_{-}\right|$, respectively, dashed black line is $\left|\phi_{+}\right|$at $K=-1373 \mathrm{rad} / \mathrm{m}$.

and $190 \mathrm{rad} / \mathrm{m}$ corresponding to 5.2 and $3.3 \mathrm{~cm}$, respectively, vertical scale variations. These can also be observed in the raw data of Figs. 7 and 8 . However over much of the range of $k_{z}$ there is more of an agreement between $\left|\phi_{+}\left(K, k_{z}\right)\right|$ and $\left|\phi_{-}\left(-K, k_{z}\right)\right|$ than between $\left|\phi_{+}\left(K, k_{z}\right)\right|$ and $\left|\phi_{-}\left(K, k_{z}\right)\right|$. This arises since Eq. (23) does not in general hold because the conditions for Taylor's hypothesis Eq. (24) do not hold. Moreover the combination of strong (but not perfect) horizontal cylindrical symmetry along with the wavefront curvature filtering effect would result in similarity between $\left|\phi_{-}\left(-K, k_{z}\right)\right|$ and $\left|\phi_{+}\left(K, k_{z}\right)\right|$. We have also indicated in Figs. 10 and 11 the Fresnel wave number $k_{F}=\pi / \sqrt{r_{0} \lambda}$ by the vertical arrow. In Fig. 11 in which there is a larger regime of wave numbers less than $k_{z}$ there does appear to be somewhat closer agreement between $\left|\phi_{-}\left(K, k_{z}\right)\right|$ and $\left|\phi_{+}\left(-K, k_{z}\right)\right|$ indicating that Taylor's hypothesis may be valid there. Figure 12 is an expanded version of $k_{z}$ of Fig. 11 in the region of this agreement.
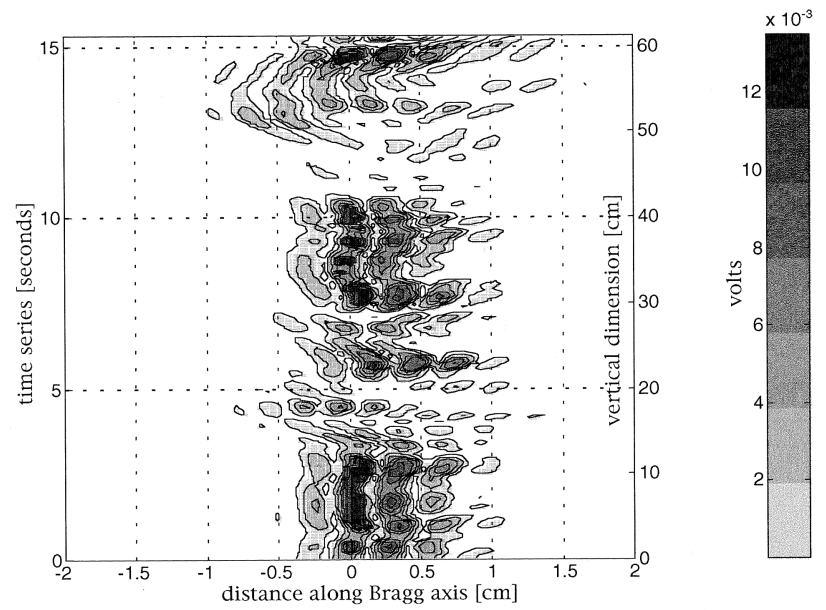

FIG. 13. Contour plot of the magnitude of the scattered signal from a turbulent plume for channel 1 . 


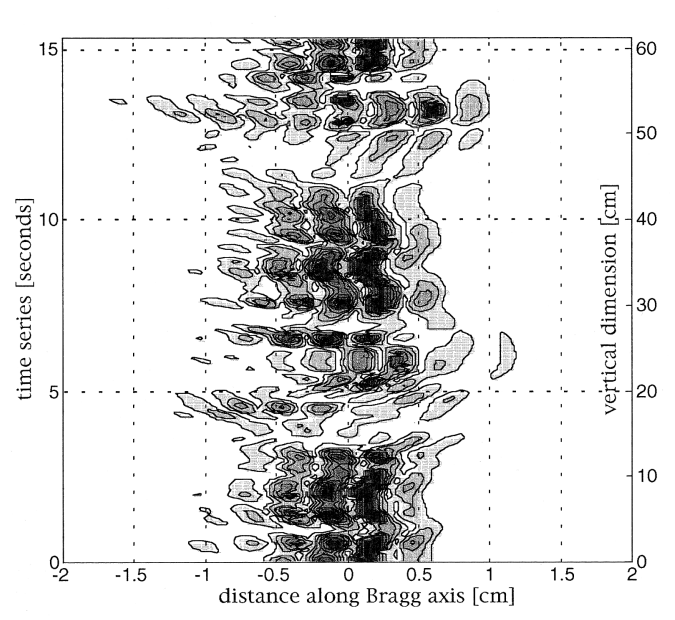

FIG. 14. Contour plot of the magnitude of the scattered signal from a turbulent plume for channel 2 .
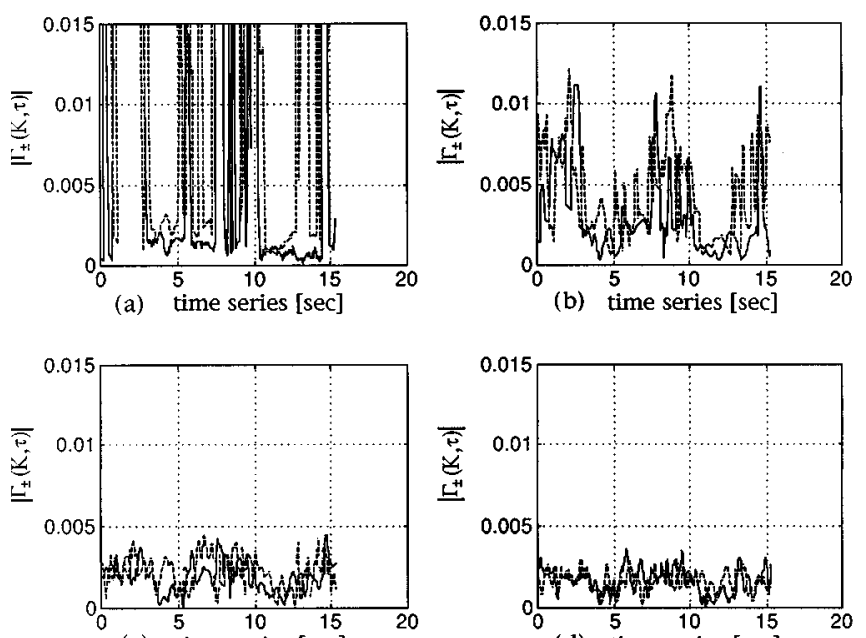

(c) time series [sec]

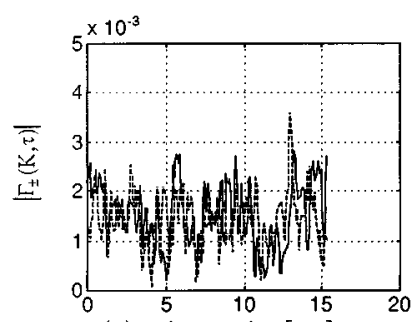

(e) time series [sec]

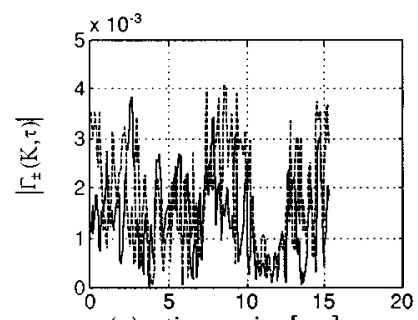

(g) time series $[\mathrm{sec}]$

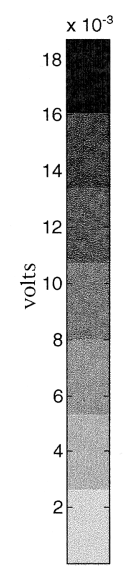

(b) time series [sec]

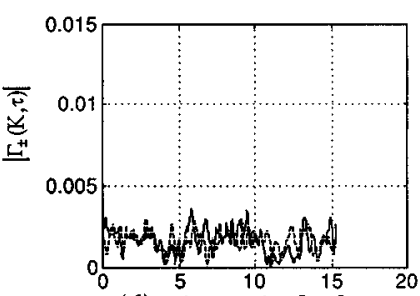

(d) time series [sec]

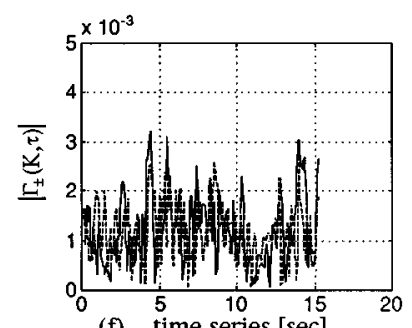

(f) time series [sec]

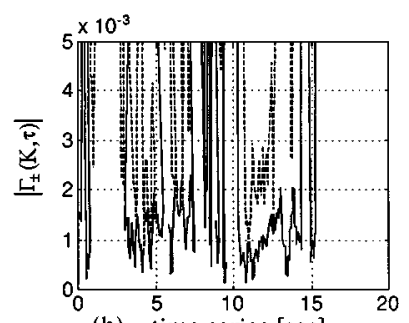

(h) time series [sec]

FIG. 15. Time series of $\left|\Gamma_{ \pm}(K, \tau)\right|$ for frequencies of (a) $117 \mathrm{kHz}$, (b) 234 $\mathrm{kHz}$, (c) $313 \mathrm{kHz}$, and (d) $430 \mathrm{kHz} . \Gamma_{+}, \Gamma_{-}$is given by solid/dashed lines, respectively. Time series of $\left|\Gamma_{ \pm}(K, \tau)\right|$ for frequencies of (e) $508 \mathrm{kHz}$, (f) $625 \mathrm{kHz},(\mathrm{g}) 703 \mathrm{kHz}$, and (h) $938 \mathrm{kHz} . \Gamma_{+}, \Gamma_{-}$is given by solid/dashed lines, respectively.
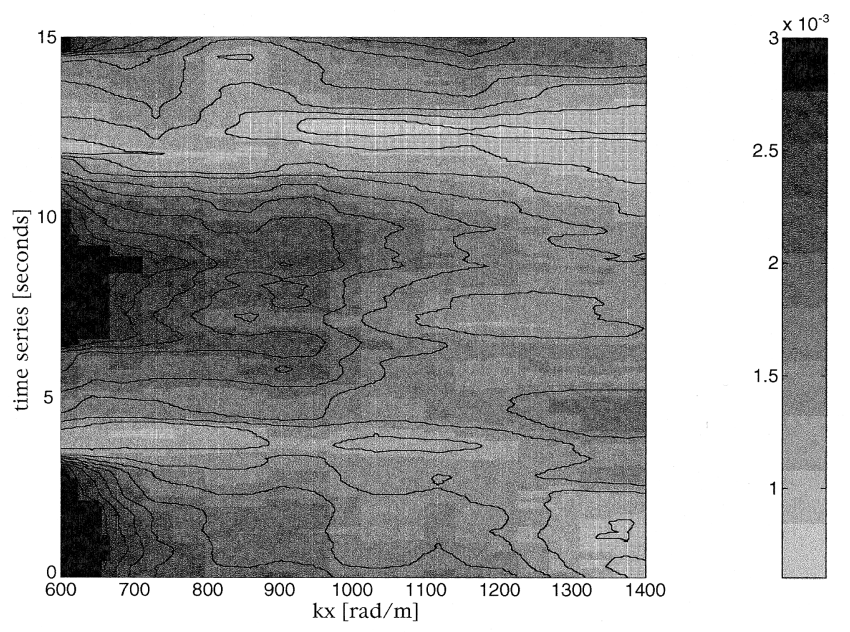

FIG. 16. Contour plot of $\Psi_{+}=\left\langle\Gamma_{+} \Gamma_{+}^{*}\right\rangle$ for turbulent plume.

\section{B. Turbulent plume}

In Figs. 13 and 14 analogous to Figs. 7 and 8 are the raw magnitude of the scattered pressure fields for channels 1 and 2 for the turbulent plume case. Note that the signal appears as a series of bursts or intense periods of duration of order 3 $\mathrm{s}$. The same type of mirror image asymmetry is also noted in these figures as in Figs. 7 and 8. Note the similarity of features in the two figures between the times of $\tau=0$ to $\tau=3 \mathrm{~s}$ and $\tau=6$ to $\tau=10 \mathrm{~s}$. In Fig. 15(a) $-(\mathrm{h})$ time series of $\Gamma_{ \pm}$are presented for this case. Note the general similarity of the two channels over the entire Bragg wave-number bandwidth. This suggests that the two fields can be considered as random realizations of the same process and an assumption of local stationarity and homogeneity is reasonable and thus that relationship (25) can be used. We calculate the filtered spectrum from $\Psi_{ \pm}=\left\langle\Gamma_{ \pm}(K, \tau) \Gamma_{ \pm}^{*}(K, \tau)\right\rangle$ where the average is taken over time. We use 151 points corresponding to approximately $2.5 \mathrm{~s}$. Equation (25) results in for the homogeneous stationary case

$$
\Psi_{+}=\Psi_{-} .
$$

In Figs. 16 and 17 we present a contour plot of $\Psi_{ \pm}$versus Bragg wave number and time. In Figs. 18(a)-(d) through we
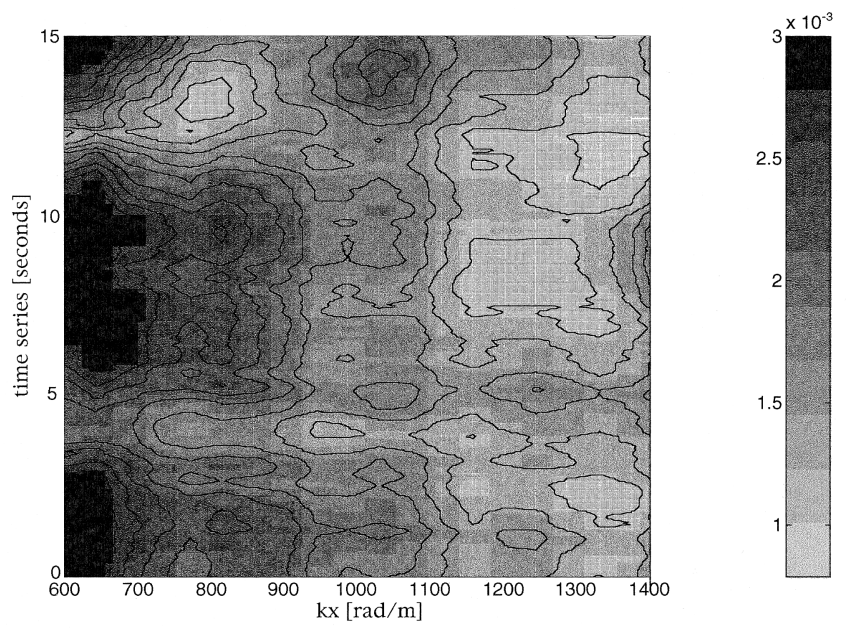

FIG. 17. Contour plot of $\Psi_{-}=\left\langle\Gamma_{-} \Gamma_{-}^{*}\right\rangle$ for turbulent plume. 
plot four curves which are "cuts" through the contour plots at different Bragg wave numbers. Note in these figures the two features seen in the time series from $\tau=0$ to $\tau=3$ and from $\tau=6$ to $\tau=10$. These features are stronger and much more broad bandwidth than the structure of the spectra at other times. This intermittency and broad bandwidth is an expected character of turbulent flow. The single scattering angle experiment does not in general resolve all the scales of the turbulent field. Our next set of experiments will be directed at this problem. Further discussion on this will be given in the summary and conclusion section. To examine the agreement of the curves and see how well (26) is satisfied we plot the fractional difference between the two spectra in Fig. 19. Equation (26) is in general well satisfied except during time period $\tau=12$ to $14 \mathrm{~s}$, which from Figs. 16 and 17 and Figs. 13 and 14 are periods of low intensity scattering. It should also be noted that there are features which clearly occur in one channel and not the other. In Fig. 17 there is a circular symmetric feature centered at $\tau=14 \mathrm{~s}$ and $K=1050$ $\mathrm{rad} / \mathrm{m}$. No such feature is present in Fig. 16. In addition the feature present centered at $\tau=13 \mathrm{~s}$ and $K=650 \mathrm{rad} / \mathrm{m}$ in Fig. 17 appears to be displaced upward above $\tau=14 \mathrm{~s}$ (and $K=650 \mathrm{rad} / \mathrm{m}$ ) in Fig. 16. In general, agreement from between $\Psi_{ \pm}$is worse at the beginning and end of the data sets. This effect is attributed to the displacement of feature effect illustrated in Fig. 4 and also noted in the unstable nonturbulent plume case.

\section{SUMMARY AND CONCLUSIONS}

The experiment discussed in this manuscript was designed to test the application of the far-field approximation to the case of scattering from unstable nonturbulent and turbulent thermally driven buoyant plumes. Unlike the laminar plume discussed in an earlier paper ${ }^{23}$ where the scattering field is two dimensional, in these cases the scattering field is a continuous distribution of three-dimensional scattering features where the vertical length scales of medium variability is of order the Fresnel radius. This has required the inclusion
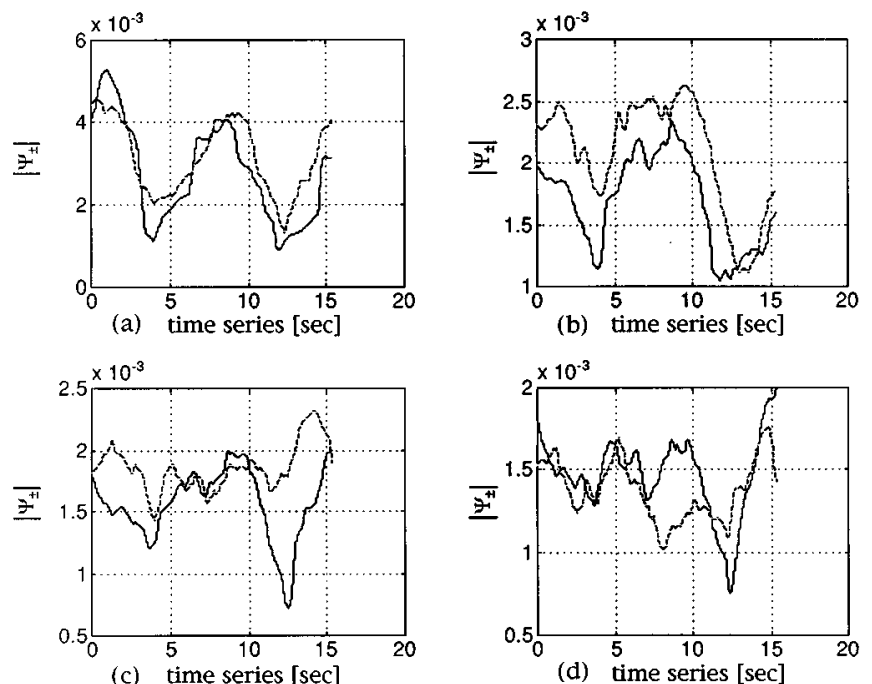

FIG. 18. Time series spectra for $\left|\Psi_{+}\right|$(solid) and $\left|\Psi_{-}\right|$(dashed) for $K=$ (a) $700 \mathrm{rad} / \mathrm{m}$, (b) $900 \mathrm{rad} / \mathrm{m}$, (c) $1100 \mathrm{rad} / \mathrm{m}$, and (d) $1300 \mathrm{rad} / \mathrm{m}$.
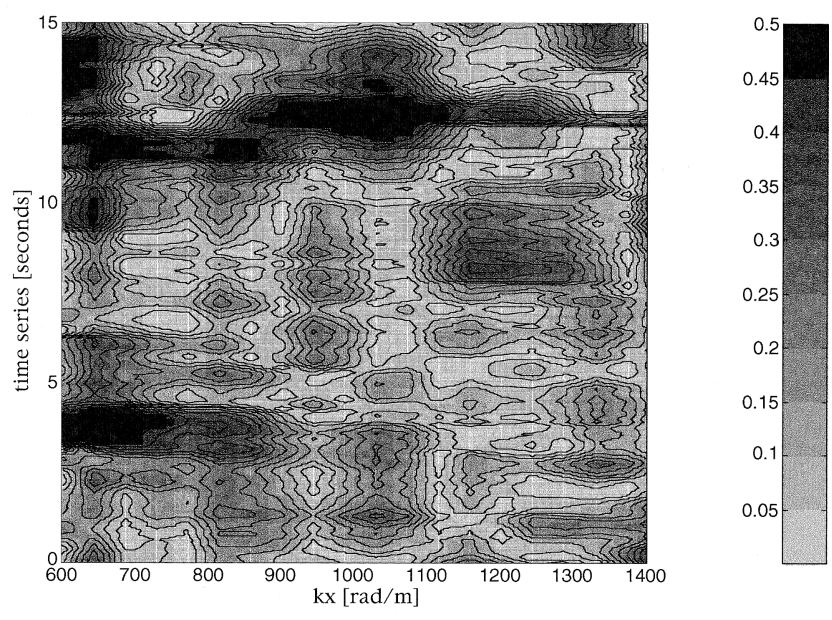

FIG. 19. Contour plot of $\frac{1}{2}\left(\Psi_{+}-\Psi_{-}\right) /\left(\Psi_{+}+\Psi_{-}\right)$.

of wavefront curvature terms in the far-field expansion of phase term in the Green's function solution. Thus the farfield approximation is insufficient to adequately describe the scattering process. A two channel bistatic scattering geometry with oppositely directed scattering wave vectors was used to observe and quantify the wavefront curvature effect. The results of the raw acoustic signals from an unstable nonturbulent plume confirmed the failure of the far-field approximation. Time series contour plots of the scattered pressure field did not indicate the mirror image symmetry which would be valid in the far-field approximation. By calculating the transfer function of the scattering process for the measurements made in the plus and minus directions, $\Gamma_{+}$and $\Gamma_{-}$, and comparing the time series at selected acoustic frequencies, the data show that $\left|\Gamma_{+}\right| \neq\left|\Gamma_{-}\right|$, thus further indication that the far-field approximation is invalid for the nonturbulent unstable plume case. It is interesting to note the qualitative similar periodicity in $\left|\Gamma_{+}\right|$and $\left|\Gamma_{-}\right|$. However the time lag shows that when one channel is measuring a maximum the other is at a minimum. This is indicative of the interaction between the wavefront curvature and the vertical length scales present in the unstable plume. Thus, far-field correction terms must be included to describe the scattering process.

The presence of wavefront curvature terms however, complicates the formerly simple relationship between the complex acoustic field and the spatial Fourier transform of the scattering field for far-field incident plane waves. This complication can be remedied however, when the scattering field satisfies Taylor's hypothesis, the plume rises with a uniform vertical velocity $w$, i.e., $T(x, y, z, \tau)=T(x, y, z$ $-w \tau)$. This assumption immediately led to the recovery of the two component three-dimensional Fourier transform of the scattering field from the acoustic data. The result predicted that $\left|\phi_{+}\left(K, k_{z}\right)\right|=\left|\phi_{-}\left(-K, k_{z}\right)\right|$, i.e., the Fourier transform of the temperature field, $\phi$, measured in the plus direction equal the negative wave-number spectrum of $\phi$ when measured in the minus direction. Note in Fig. 11 the agreement in $\left|\phi_{+}\left(K, k_{z}\right)\right|$ and $\left|\phi_{-}\left(-K, k_{z}\right)\right|$ at lower $k_{z}$ values, however at larger $k_{z}$ values the agreement breaks down. This result indicates the length scales over which Taylor's 
hypothesis may hold are $k_{z}\left\langle 200-300 \mathrm{rad} / \mathrm{m}\right.$, or $\left.l_{z}=2 \pi / k_{z}\right\rangle$ $2-3 \mathrm{~cm}$. Figure 12 indicates that the large scale vertical variability better satisfies Taylor's hypothesis than the smaller scale variability.

Scattering from a turbulent plume using the oppositely directed scattering geometry was also examined. As with the unstable nonturbulent plume, time series data of the scattered pressure field from the turbulent plume showed the same qualitative asymmetry when viewed from opposite directions. However, unlike the unstable plume, time series comparisons of the transfer functions for the turbulent plume were in good agreement. This results from the statistical equivalence of the turbulent plume within the scattering volume when viewed from opposing directions.

For the turbulent plume, the assumption of local homogeneity and stationarity was used to develop a wave-number spectrum of the scattering field, $\Psi$, which resulted in predicting equal spectra for oppositely directed scattering geometry measurements. The fractional difference between $\Psi_{+}$and $\Psi_{\text {- showed that for instances away from the initial and final }}$ conditions of the experiment, agreement from 5\% to $25 \%$ over the bandwidth of the measurement. This technique remains to be more fully utilized in the common scattering direction experiment to better determine the wave-number spectrum of the scattering field over a broader bandwidth.

Application of this technique to remotely monitor seafloor vents and plumes presents some difficulties. The theoretical development described in this paper requires the use of the weak scattering approximation. Acoustic imaging measurements of hydrothermal plumes conducted by Rona et al. ${ }^{29}$ had plume temperatures of $350{ }^{\circ} \mathrm{C}$ and equivalent diameters of $5.3 \mathrm{~cm}$. Using these values in Eq. (7), the required condition for weak scattering theory, the inequality is clearly violated. Thus even in the absence of particulates the complex receive acoustic field is no longer simply related to the temperature field of the scattering volume. Rona et al. stated that the acoustic imaging used was based on backscatter due to metallic mineral particles precipitated in the plume. Whether the dominate scattering mechanism was particulates or thermally induced index of refraction changes, it is reasonable to state that weak scattering theory may not be satisfied. The use of Taylor's hypothesis to describe vertical advection is valid provided the time scale of vertical variability is much greater than the ratio of the Fresnel radius to the vertical velocity of the plume, [Eq. (24)]. Rona et al. quote an initial plume velocity of $1 \mathrm{~m} / \mathrm{s}$, at $500 \mathrm{kHz}$ and a plume receiver of range of $5 \mathrm{~m}$ yields a limiting time scale of $0.1 \mathrm{~s}$. Thus this method could be used to infer vertical variability of such sea floor vents. Apart from examining seafloor vents and plumes, application of the methods and techniques developed can be applied to examine turbulence found in the ocean. Particular use can be made, as will be discussed in a future paper, on using multiple bistatic scattering measurements made in a common scattering direction.

\section{ACKNOWLEDGMENTS}

This work has been supported by the Office of Naval Research, Code 3210A, Dr. Jeff Simmen, Program Manager, and by the Naval Undersea Warfare Center, Independent Research Program, Dr. Stuart Dickenson, Program Manager. The authors would also like to thank Dr. Steve Letcher, Diane Medeiros, Peter Hebda, and Dave Notarianni for their assistance.

\section{APPENDIX}

Expansion of $\left|\mathbf{r}-\mathbf{r}^{\prime}\right|$ in terms of integration variables up to fourth order gives

$$
\begin{aligned}
\left|\mathbf{r}+\mathbf{r}^{\prime}\right|= & 2 r_{0}-2 x \sin \frac{\theta}{2}+\frac{z^{2}}{r_{0}}+\frac{x^{2}}{r_{0}} \cos ^{2} \frac{\theta}{2}+\frac{y^{2}}{r_{0}} \sin ^{2} \frac{\theta}{2}+\frac{x z^{2}}{r_{0}^{2}} \sin \frac{\theta}{2}+\frac{x^{3}}{r_{0}^{2}} \sin \frac{\theta}{2} \cos ^{2} \frac{\theta}{2}+\frac{x y^{2}}{r_{0}^{2}} \sin ^{3} \frac{\theta}{2}-\frac{z^{4}}{4 r_{0}^{3}}-\frac{z^{2}}{2 r_{0}^{3}}\left(x^{2}-2 y^{2}\right) \\
& \times \cos ^{2} \frac{\theta}{2}-\frac{z^{2}}{2 r_{0}^{3}}\left(y^{2}-2 x^{2}\right) \sin ^{2} \frac{\theta}{2}-\frac{x^{2}}{4 r_{0}^{3}}\left(x^{2}+4 y^{2}\right) \cos ^{4} \frac{\theta}{2}-\frac{y^{2}}{4 r_{0}^{3}}\left(y^{2}+4 x^{2}\right) \sin ^{4} \frac{\theta}{2} \\
& -\left(\frac{x^{4}}{r_{0}^{3}}-\frac{5}{2} \frac{x^{2} y^{2}}{r_{0}^{3}}+\frac{y^{4}}{r_{0}^{3}}\right) \sin ^{2} \frac{\theta}{2} \cos ^{2} \frac{\theta}{2}+\cdots .
\end{aligned}
$$

A term-by-term evaluation of $k\left|\mathbf{r}+\mathbf{r}^{\prime}\right|$ using the above expansion and $k=1380 \mathrm{rad} / \mathrm{m}$ (corresponding to the $6-\mathrm{dB}$ power down point), $r_{0}=25.0 \mathrm{~cm}, \theta=30^{\circ}, x=y=0.4 \mathrm{~cm}$, and $z=3.0$ $\mathrm{cm}$. Only the first three terms on the right-hand side of (A1) are of order or greater than one. The parameters used for the horizontal variability of the plume are determined by the $e$-folding value of the laminar plume ${ }^{23}$ coupled with time series temperature data taken at a point located directly above the scattering volume and simultaneously with the acoustic data. The vertical extent of the scattering field is limited by the source/receiver beam pattern and determined using the $6 \mathrm{~dB}$ down point at the lower limiting frequency.

\footnotetext{
${ }^{1}$ J. R. Proni and J. R. Apel, "'On the Use of High-Frequency Acoustics for the Study of Internal Waves and Microstructure,' J. Geophys. Res. 80, 1147-1151 (1975).

${ }^{2}$ S. A. Thorpe and J. M. Brubaker, "'Observations of sound reflection by temperature microstructure," 'Limnol. Oceanogr. 28, 601-613 (1983).

${ }^{3}$ F. R. Hess and M. H. Orr, "A Towed Multifrequency H.F. Sonar System for Scattering and Ocean Dynamics Study,' Rep. WHOI-79-76, Woods Hole Oceanogr. Inst., 1979.

${ }^{4}$ L. R. Haury, P. H. Wiebe, M. H. Orr, and M. G. Briscoe, "Tidally Generated High-Frequency Internal Wave Packets and their Effects on Plankton in Massachusetts Bay," J. Mar. Res. 41, 65-112 (1983).
} 
${ }^{5}$ L. Goodman, "Acoustic Scattering From Ocean Microstructure," J. Geophys. Res. 95, 11 557-11 573 (1990).

${ }^{6}$ W. Baerg and W. H. Schwarz, "Measurements of the Scattering of Sound from Turbulence,', J. Acoust. Soc. Am. 39, 1125-1132 (1966).

${ }^{7}$ M. J. Lighthill, "'On The Energy Scattered From The Interaction Of Turbulence With Sound Or Shock Waves,' Proc. Cambridge Philos. Soc. 49, 531-551 (1953)

${ }^{8}$ R. H. Kraichnan, "The Scattering of Sound in a Turbulent Medium,' J. Acoust. Soc. Am. 25, 1096-1104 (1953).

${ }^{9}$ G. K. Batchelor, in Symposium on Naval Hydrodynamics, edited by F. S. Sherman (National Academy of Science-National Research Council, Washington, DC, 1957).

${ }^{10} \mathrm{G}$. K. Batchelor, The Theory of Homogeneous Turbulence (Cambridge U.P., Cambridge, England, 1953).

${ }^{11}$ A. Brandt, "Acoustic Return From Density Fluctuations in Turbulent Jets,' IEEE Ocean 8-12 (1975).

${ }^{12}$ M. S. Korman and R. T. Beyer, "The scattering of turbulence in water," J. Acoust. Soc. Am. 67, 1980-1987 (1980).

${ }^{13}$ A. Ishimaru, Wave Propagation and Scattering in Random Medium (Academic, New York, 1978), Vol. 2, Chap. 16.

${ }^{14}$ J. A. Campbell and R. C. Waag, "Normalization of ultrasonic scattering measurements to obtain average differential scattering cross sections for tissues," J. Acoust. Soc. Am. 74, 393-399 (1983).

${ }^{15}$ J. A. Campbell and R. C. Waag, "Measurements of calf liver ultrasonic differential and total scattering cross sections," J. Acoust. Soc. Am. 75, 603-611 (1984).

${ }^{16}$ D. Nicholas, C. R. Hill, and P. K. Nasseri, "Evaluation of Backscattering Coefficients for Excised Human Tissue: Principles and Techniques,' U1trasound Med. Biol. 8, 7-15 (1982).

${ }^{17}$ D. Nicholas, "Evaluation of Backscattering Coefficients for Excised Human Tissue: Results, Interpretation, and Associated Measurements,' Ultrasound Med. Biol. 8, 17-28 (1982).
${ }^{18}$ D. K. Nassiri and C. R. Hill, "The differential and total acoustic scattering cross sections of some human and animal tissues," J. Acoust. Soc. Am. 79, 2034-2047 (1986).

${ }^{19}$ D. K. Nassiri and C. R. Hill, "The use of acoustic scattering measurements to estimate structural parameters of human and animal tissues," J. Acoust. Soc. Am. 79, 2048-2054 (1986).

${ }^{20}$ R. C. Waag, D. Dalecki, and W. A. Smith, "Estimates of wavefront distortion from measurements of scattering by model random media and calf liver," J. Acoust. Soc. Am. 85, 406-415 (1989).

${ }^{21}$ R. C. Waag, D. Dalecki, and P. E. Christopher, "Spectral power determination of compressibility and density variations in model media and calf liver using ultrasound," J. Acoust. Soc. Am. 85, 423-431 (1989).

${ }^{22} \mathrm{~A}$. Witten and J. Tuggle, "A practical approach to ultrasonic imaging using diffraction tomography," J. Acoust. Soc. Am. 83, 1645-1652 (1988).

${ }^{23}$ L. Goodman, J. Oeschger, and D. Szargowicz, "Ocean acoustics turbulence study: Acoustic scattering from a buoyant axisymmetric plume," J. Acoust. Soc. Am. 91, 3212-3227 (1992).

${ }^{24}$ P. M. Morse and K. U. Ingard, Theoretical Acoustics (McGraw-Hill, New York, 1968).

${ }^{25}$ V. I. Tatarski, Wave Propagation in a Turbulent Medium (McGraw-Hill, New York, 1961).

${ }^{26}$ A. Ishimaru, Wave Propagation and Scattering in Random Medium (Academic, New York, 1978), Vol. 1, Chap. 2.

${ }^{27}$ L. E. Kinsler, A. R. Frey, A. B. Coppens, and J. V. Sanders, Fundamentals of Acoustics (Wiley, New York, 1982).

${ }^{28}$ J. O. Hinze, Turbulence (McGraw-Hill, New York, 1959).

${ }^{29}$ P. A. Rona, D. R. Palmer, C. Jones, D. A. Chayes, M. Czarnecki, E. W. Carey, and J. C. Guerrero, "Acoustic imaging of hydrothermal plumes, East Pacific Rise, $21^{\circ} \mathrm{N}, 109^{\circ} \mathrm{W}, "$ Geophys. Res. Lett. 18, 2233-2236 (1991) 